\title{
Purulent Pericarditis: Is It Really a Disease of the Past?
}

\author{
Laura Costa $^{1}$, Diana Carvalho ${ }^{2}$, Elisabete Coelho ${ }^{1}$, Dina Leal ${ }^{1}$, Luís Lencastre ${ }^{1}$ \\ ${ }^{1}$ Unidade de Cuidados Intensivos Polivalente, Hospital de Braga, Braga, Portugal \\ ${ }^{2}$ Serviço de Cardiologia, Centro Hospitalar do Baixo Vouga, Aveiro, Portugal
}

Received: 20/05/2021

Accepted: 25/05/2021

Published: $14 / 07 / 2021$

How to cite this article: Costa L, Carvalho D, Coelho E, Leal D, Lencastre L. Purulent pericarditis: is it really a disease of the past? EJCRIM 2021;8: doi:10.12890/2021_002658.

Conflicts of Interests: The authors declare there are no competing interests.

This article is licensed under a Commons Attribution Non-Commercial 4.0 License

\section{ABSTRACT}

The authors present a case of purulent pericarditis probably secondary to respiratory infection, a rare entity in the antibiotic era. Pericardial fluid analysis identified streptococci and oral anaerobes as the causative agents. A prolonged and complicated diagnostic and therapeutic course, which included a long stay in the intensive care unit, is described, and a review of purulent pericarditis provided. Pericardial effusion, particularly in the setting of concomitant respiratory infection and immunocompromise or other risk factors, should raise the suspicion of bacterial pericarditis and prompt its timely diagnosis and treatment. Purulent pericarditis can be lethal and has potentially severe complications, so adequate antimicrobial therapy and source control are key.

\section{LEARNING POINTS}

- Purulent pericarditis is a rare infection, mostly resulting from contiguous or haematogenous spread, with diagnosis often delayed because signs and symptoms are unspecific.

- Treatment must include drainage of the pericardial space, combined with systemic antibiotics.

- Prognosis is variable, depending largely on the speed of diagnosis and treatment, as death is almost certain without adequate source control.

\section{KEYWORDS}

Bacterial pericarditis, secondary pericarditis, acute mediastinitis, pericardiocentesis

\section{CASE DESCRIPTION}

We describe the case of a 54-year-old man with GOLD stage D COPD and treated pulmonary tuberculosis who presented with precordial pain, constitutional symptoms and aggravated dyspnoea. He was admitted to the ward for respiratory infection.

During hospitalization, he experienced several episodes of arrhythmia and exacerbation of his symptoms. Three days after admission, a chest CT scan identified a large pericardial effusion, extensive bronchiectasis, and a cavitated lung lesion with an air-fluid level. There was rapid evolution to obstructive shock, requiring invasive ventilation and cardiovascular support. Emergent pericardiocentesis was performed with drainage of approximately 1 litre of pus. The patient was started on piperacillin/tazobactam and vancomycin and remained dependent on inotropic/vasopressor support in the intensive care unit. After multidisciplinary discussion with cardiology and cardiac surgery, it was decided to maintain pericardial drainage/flushing and antibiotic therapy.

Following the identification of Streptococcus anginosus and Fusobacterium sp. in the pericardial fluid, the antimicrobial regimen was adjusted to penicillin $\mathrm{G}$ and clindamycin. The patient's condition evolved favourably with resolution of shock and associated dysfunction and he was extubated on the 10th day of ICU stay. However, after 1 week, he was again febrile, with elevated inflammatory parameters and cellulitis 
of the chest and abdomen. The CT scan was repeated and identified exuberant mediastinitis, with a multiloculated abscessal collection $(210 \times 190 \times 46 \mathrm{~mm})$ from the anterior mediastinum to the subdiaphragmatic planes (Figs. 1-3). The patient was immediately transferred for surgical cleaning. Antibiotic therapy was escalated to meropenem/vancomycin and maintained for 28 days after surgery. No further isolates were detected. The patient improved and was discharged to a general ward.

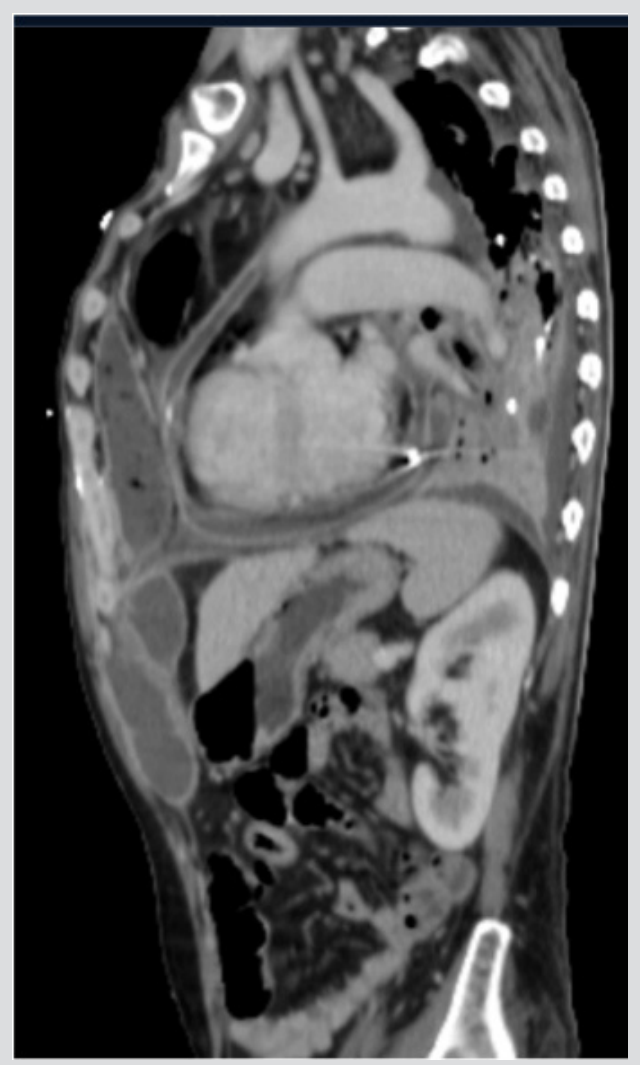

Figure 1. Sagittal view: Collection in the anterior mediastinum (pre-cardiac space) measuring $185 \times 20 \mathrm{~mm}$; collection in the subdiaphragmatic space, anteriorly to the renal left lobe measuring $60 \times 32 \mathrm{~mm}$

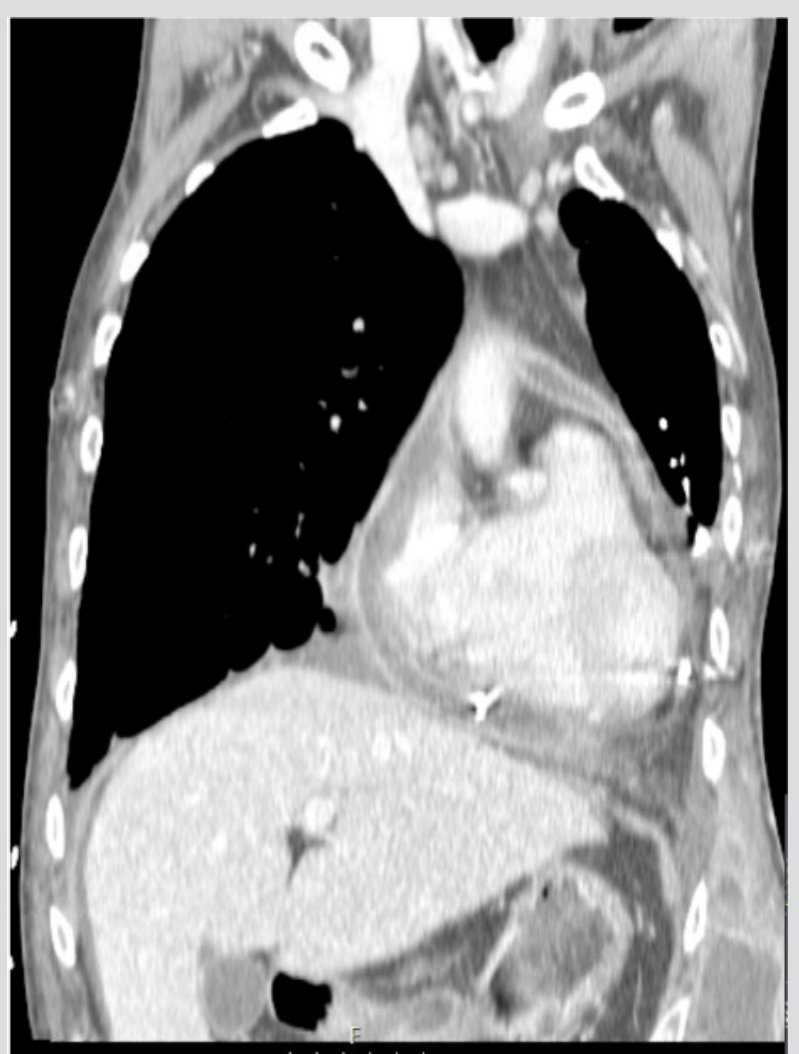

Figure 2. Coronal view: pericardial effusion $(3 \mathrm{~mm})$ with thickening of the pericardial leaflets, reflecting active inflammation. Mediastinal paratracheal, pre-vascular and paraaortic adenomegalies are also seen

\section{DISCUSSION}

Purulent pericarditis is defined as an infection in the pericardial space that produces macroscopically or microscopically purulent fluid ${ }^{[1]}$. Once a common condition, purulent pericarditis is now a rarity with an estimated incidence of 1/18,000, in part due to the availability of effective antimicrobials. Historically considered a disease of children and the young, today it is seen from the fifth decade of life ${ }^{[2]}$. Clinical manifestations are variable, and diagnosis is often delayed due to absence of the typical signs of pericarditis and attribution of the constitutional symptoms to other infections ${ }^{[2,3]}$. The diagnosis can only be confirmed by pericardiocentesis (which in such cases is also therapeutic) by means of macroscopic fluid examination, and biochemical analysis, which shows features of an exudate ${ }^{[1]}$. Although extremely rare, bacterial pericarditis may be primary. More commonly, it is secondary to another infectious process which may have an intrathoracic or subdiaphragmatic origin, or result from a perforating injury or surgery or from haematogenous spread ${ }^{[3,4]}$. The risk factors have changed over time: in the pre-antibiotic era, the condition mostly resulted from pulmonary infection, with secondary extension to the pericardium. In the post-antibiotic era, cardiac and thoracic procedures are considered the main vehicles of infection, with thoracic surgery, chronic kidney disease, immunosuppression, alcoholism and neoplasms being the identified risk factors ${ }^{[3,5]}$. The pericardial fluid should be subjected to microscopic study, direct and in culture, to screen for bacteria, fungi and acid-alcohol resistant bacilli (AARB). Streptococci used to be the most common causative agents of purulent pericarditis. However, the discovery and use of antibiotics drastically reduced their prevalence, and today we are witnessing a relative increase in Gram-negative bacteria ${ }^{[1]}$. Anaerobes are implicated in $40 \%$ of cases, predominantly oral flora. Treatment is based on adequate systemic antibiotic therapy and drainage of the pericardial space. 


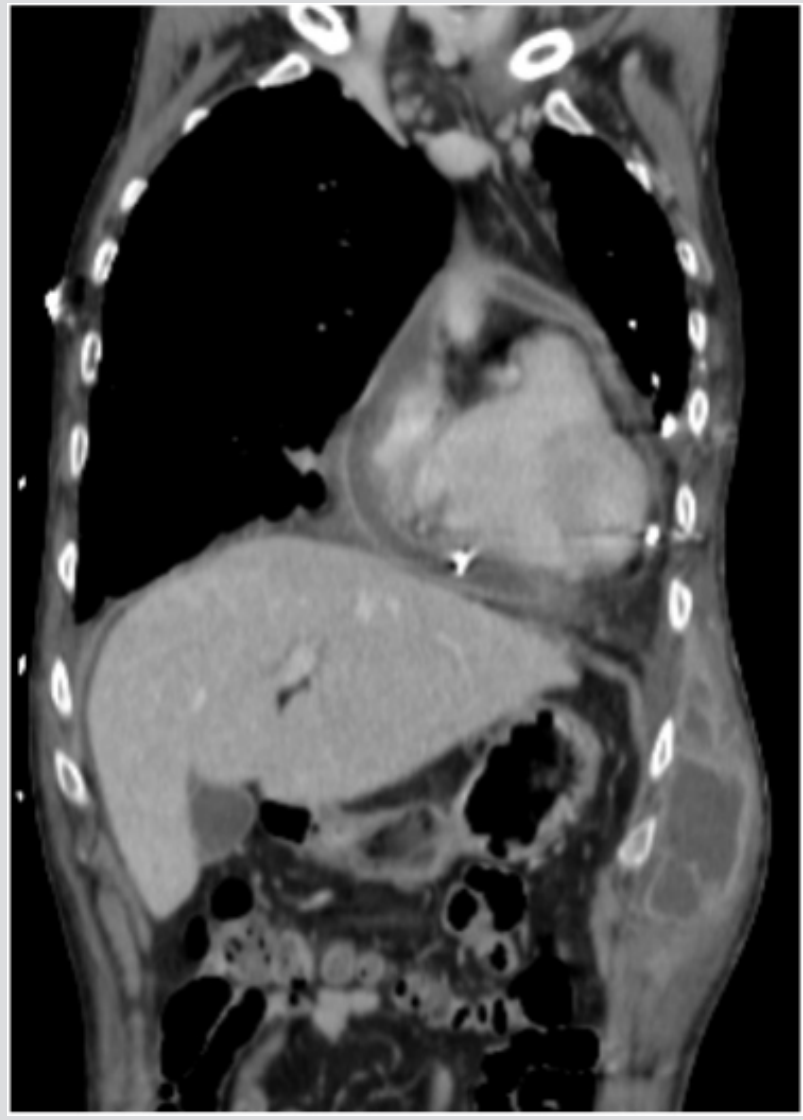

Figure 3. Coronal view: collection in subcutaneous tissue, adjacent to the left inferior costal margin

Loculation may occur with incomplete drainage, which increases the risk of constrictive pericarditis. In these cases, surgery employing a pericardial window or pericardiectomy is required. Without early treatment, mortality is close to $100 \%$, but with treatment it reduces to approximately $40 \%{ }^{[2]}$.

\section{CONCLUSION}

We describe purulent pericarditis probably secondary to pulmonary infection, that manifested with cardiac tamponade. It is a rare entity, almost a historical one, and in our case, presented with clinical, epidemiological and microbiological features that were characteristic of the pre-antibiotic era. Even today purulent pericarditis is associated with high mortality and serious complications, so early diagnosis and source control are mandatory.

\section{REFERENCES}

1. Ferreira dos Santos L, Moreira D, Ribeiro P, Rodrigues B, Correia E, Nunes L, et al. Purulent pericarditis: a rare diagnosis. Rev Port Cardiol 2013;32(9):721-727.

2. Sagristà-Sauleda J, Barrabés JA, Permanyer-Miralda G, Soler-Soler J. Purulent pericarditis: review of a 20-year experience in a general hospital. J Am Coll Cardiol 1993;22(6):1661-1665. doi: 10.1016/0735-1097(93)90592-O

3. Pankuweit S, Ristić AD, Seferović PM, Maisch B. Bacterial pericarditis: diagnosis and management. Am J Cardiovasc Drugs 2005;5(2):103-112. doi: 10.2165/00129784200505020-00004

4. Sagristà-Sauleda J, Mercé J, Permanyer-Miralda G, Soler-Soler J. Clinical clues to the causes of large pericardial effusions. Am J Med 2000;109(2):95-101. doi: 10.1016/ s0002-9343(00)00459-9

5. Kabbani SS, LeWinter MM. Pericardial diseases. Curr Treat Options Cardiovasc Med 2002;4(6):487-495. doi: 10.1007/s11936-002-0042-0 